\title{
Evaluation of Herbal Formulations on Fungal Pathogens of Plants: A Case Study
}

\author{
Sumangala Bhat K*, Kalpana P, Aishwarya T, Krithika G \\ Dextrose Technologies Pvt. Ltd., Bengaluru, India \\ *Corresponding Author: Sumangala Bhat K, Dextrose Technologies Pvt. Ltd., Bengaluru, India.
}

Received: November 25, 2019; Published: November 29, 2019

DOI: $10.31080 /$ ASAG.2019.03.0734

\begin{abstract}
Plant pathogens and pests are the major challenges in crop production since ancient times and continue to haunt the agricultural industry even today. Fungal pathogens take a heavy toll on crop yield across all crop lants world over. Chemical fungicides dominate the disease management strategies in agriculture, though many of them are known pollutants of the environment at large. Plantderived fungicides have been explored and evolved as a safer alternative during the recent past, and still, more research needs to be done on this domain. We present the results of the in vitro evaluation of plant essential oil-based antifungal formulations, WiltProof and Fungiproof developed by our company on Fusarium solani and Helminthosporium sp. isolated from infected plant materials. Trials on the efficacies of the two formulations on PDA medium in terms of colony diameter has demonstrated fungistatic effects of both formulations on the fungi tested with FungiProof showing higher activity. In situ trials using bell pepper and $F$. solani as a model system for investigating the infection cycle and influence of the intervention by the two products in the laboratory also confirmed the above conclusion on relative efficacies of the two products.
\end{abstract}

Keywords: Essential Oils; Fusarium solani; Helminthosporium sp.; Fungistatic Efficacy

\section{Introduction}

Agriculture is one of the oldest occupations of human beings, got evolved from time to time. Agriculture turned out to be technology-driven during the last few decades and still the gap between the demand and supply of food is widening across regional and global levels [1-3]. Plant diseases play a crucial role in determining crop yield. Fungal diseases rank among the top causes of crop loss [4], affecting all types of crop plants. Fungicides are arguably a predominant input to agro-ecosystems. Regular application of fungicides poses a potential risk to the environment with multifaceted impact [5] on soil, water, and air affecting a wide range of organisms. Hence, there is an increasing demand to reduce the use of chemical fungicides and the development of eco-friendly and effective products for managing fungal pathogens affecting crop plants. Essential oils of plants are a rich source of secondary metabolites, particularly concerned with plant defense against pathogens and pests. Eugenol is a naturally occurring phenolic compound and a major component of essential oils extracted from plants including clove, cinnamon, basil, and nutmeg [6]. This compound exhibits many biological activities such as analgesic, antimicrobial, antifungal, anti-inflammatory, antioxidant, anti-carcinogenic and anti-mutagenic [7]. Therefore, these oils can be a potential source of raw materials for the development of eco-friendly products to tackle plant fungal pathogens. In the current, study we have attempted a comparative evaluation of the antifungal efficacy of two herbal formulations namely, WiltProof and FungiProof, developed by Dextrose Technologies Pvt. Ltd., India.

\section{Materials and Methods \\ Collection of samples}

Current study was designed to evaluate the efficacy of two antifungal herbal formulations Wiltproof and Fungiproof developed by the company on plant pathogenic fungi. The pathogens selected for the study were Fusarium solani. and Helminthosporium sp. The fungal cultures were originally isolated from infected bell pepper fruits and bamboo leaves respectively. The isolates were cultured on V8 agar medium and incubated at $27-28^{\circ} \mathrm{C}$.

In vitro antifungal efficacy assay

From the seven days old culture, agar plug of 6-mm diameter containing a sporulated colony of each fungus was obtained and placed on V8 agar in plates containing different concentrations of WiltProof and FungiProof $(0.5,1.0,1.5 \mu \mathrm{l} / \mathrm{ml})$. For positive control, the agar plate without any treatment was inoculated with the test organisms. Plates in two replicates were used for each treatment in the assay. All plates were incubated at $27-28^{\circ} \mathrm{C}$ for 4 days to compare the growth of the fungal mycelia. The mycelial growth in the presence and absence of WiltProof and FungiProof at different concentrations were measured. The antifungal activity was determined by measuring the colony diameter. Percentage efficacy of WiltProof and FungiProof was determined using the relationship between colony diameter of the control and the test [8], using the formula:

\section{Percentage Efficacy $=[(\mathrm{Cdc}-\mathrm{Cdt}) / \mathrm{Cdc}]^{*} 100$}

where Cdc is Colony diameter of the control and Cdt is Colony diameter of the test. 


\section{In situ antifungal efficacy assay}

F. solani spore suspension was prepared from 7days old broth culture by filtering the culture broth through filter paper. Using a Haemocytometer, the number of spores in the suspension was estimated to be approximately $10^{5}$ spores per ml. Freshly harvested Bell peppers were obtained from agricultural fields of IIHR, Hessarghatta, India and surface sterilized. Wounds of $5 \times 5 \mathrm{~mm}$ were inflicted on the surface of the bell pepper with a sterile surgical blade and prepared for inoculation. The wounded bell peppers were sprayed with a $1 \% \mathrm{v} / \mathrm{v}$ solution of WiltProof and FungiProof in sterile water $[9,10]$. The positive control was sprayed with Bavastin $1 \% \mathrm{w} / \mathrm{v}$. The samples were allowed to dry at room temperature. The wounds of the treated bell peppers were then inoculated with $20 \mu \mathrm{l}$ of $F$. solani spore suspension. The negative control consisted of wounded fruit without any treatment, either antifungal agent or inoculation by the pathogen. Each of the treatment was carried out in 10 replicates. The entire process was carried out in a sterile zone in a Laminar Air Flow chamber and the samples were then placed in sterile polythene bags lined with moist sterile tissue paper. The samples were incubated at room temperature for 20 days, with regular monitoring.

\section{Results and Discussion}

Percentage efficacy of Wiltproof and Fungiproof

The mycelial growths of both F. solani and Helminthosporium sp. were most sensitive to Fungiproof (Figure 1) and moderately sensitive to Wiltproof (Figure 2). The percentage efficacy of the products on mycelial radial growth was measured and compared (Table 1). As the percentage efficacy of FungiProof was found to be higher than that of WiltProof, mycelial growth of $F$. solani and Helminthosporium sp. was more effectively inhibited by FungiProof in comparison with WiltProof.

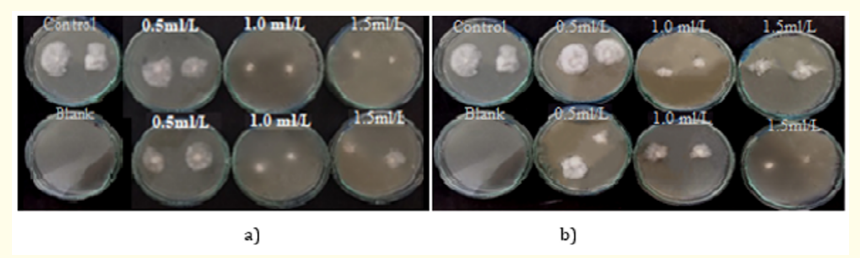

Figure 1: Effect of a) FungiProof and b) WiltProof on Hyphal growth of Fusarium solani in PDA medium. Photogaphs show growth of $F$. solani in the absence (control) and presence of different concentrations $(0.5,1.0$ and $1.5 \mathrm{ml} / \mathrm{L})$ after of 4 days.

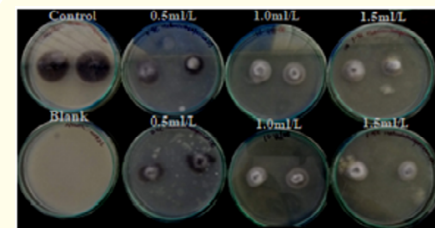

a)

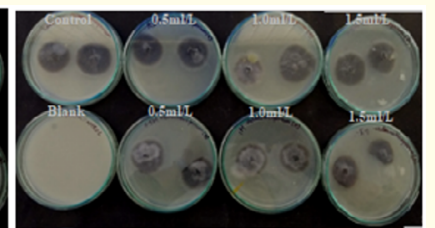

b)
Figure 2: Effect of a) FungiProof and b) WiltProof on Hyphal growth of Helminthosporium sp. in PDA medium. Photogaphs show growth of $F$. solani in the absence (control) and presence of different concentrations $(0.5,1.0$ and $1.5 \mathrm{ml} / \mathrm{L})$ after of 4 days.

\begin{tabular}{|c|c|c|c|c|c|c|}
\hline \multirow[t]{2}{*}{ Test Organism } & \multicolumn{3}{|c|}{$\begin{array}{c}\% \text { Efficacy of WiltProof at different } \\
\text { concentrations }\end{array}$} & \multicolumn{3}{|c|}{$\begin{array}{l}\% \text { Efficacy of FungiProof at different } \\
\text { concentrations }\end{array}$} \\
\hline & $0.5 \mathrm{ml} / \mathrm{L}$ & $1 \mathrm{ml} / \mathrm{L}$ & $1.5 \mathrm{ml} / \mathrm{L}$ & $0.5 \mathrm{ml} / \mathrm{L}$ & $1 \mathrm{ml} / \mathrm{L}$ & $1.5 \mathrm{ml} / \mathrm{L}$ \\
\hline Fusarium solani & $15.97 \%$ & $50.82 \%$ & $64.33 \%$ & $48.28 \%$ & $52.92 \%$ & $73.06 \%$ \\
\hline Helminthosporium sp. & $16.75 \%$ & $17.44 \%$ & $35.78 \%$ & $21.92 \%$ & $38.1 \%$ & $41.8 \%$ \\
\hline
\end{tabular}

Table 1: Fungistatic efficacy of WiltProof and FungiProof on F. solani and Helminthosporium sp. under in vitro condition, based on colony diameter on $4^{\text {th }}$ day of incubation.

\section{In situ assay of antifungal efficacy on bell pepper}

Typical lesions of Fusarium could be observed after 20 days of incubation of the control set of bell pepper samples exposed to spore suspension under laboratory conditions (Figure 3). All of the control samples developed the lesions followed by the decay of the fruit. Among the test samples, WiltProof treatment showed 30\% of the fruits developing lesions at a timeline similar to that of the control fruits (Figure 3a). However, none of the fruits treated with FungiProof and the positive control Bavistin developed any symptom of infection (Figure 3b, 3c).

The in vitro and in situ evaluation of the herbal formulations have confirmed their antifungal efficacy, FungiProof being more effective than WiltProof. The efficacy of FungiProof is on par with the popular chemical fungicide Bavistin. The antifungal and fungistatic activity has been reported for eugenol, one of the predomi- nant compounds present in most of the plant essential oils. The mechanism of action of eugenol is attributed to the alteration of permeability and fluidity of the fungal membrane [6]. The lack of growth of F. solani in treated samples of bell pepper has confirmed the prevention of fungal growth within the experimentally infected wounds of the fruits under laboratory conditions.

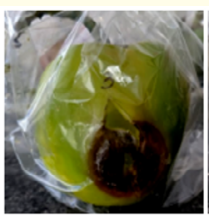

a)

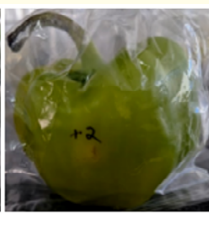

b)

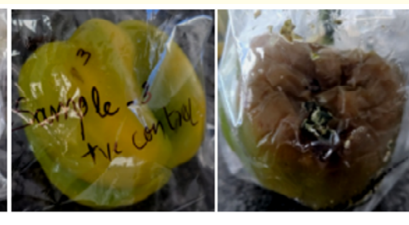

c)
Figure 3: In situ bioassay for evaluation of fungistatic efficacy of a) WiltProof and b) FungiProof and c) Bavistin on bell peppers experimentally infected with $F$. solani spore suspension alnong with the untreared control d). 


\begin{tabular}{|l|c|}
\hline \multicolumn{1}{|c|}{ Treatment } & Results \\
\hline Wilt Proof & $30 \%$ fruits infected \\
\hline Fungi Proof & $0 \%$ fruits infected \\
\hline Bavastin (+ve control) & $0 \%$ fruits infected \\
\hline No treatment (-ve control) & $100 \%$ fruits infected \\
\hline
\end{tabular}

Table 2: In situ evaluation of fungistatic efficacy of Wilt Proof and Fungi Proof on bell pepper experimentally infected with $F$. solani.

\section{Conclusion}

The investigation has confirmed the efficacy of both WiltProof and FungiProof on F. sloani and Helminthosporium sp., with FungiProof showing a higher level of efficacy at par with commercial fungicide, Bavistin. The activity of eugenol present in the essential oils is attributed to the antifungal activity of the formulations. The synergistic activity of different secondary metabolites, in the two test formulations, can be related to the difference in the extent of activity of the two products.

\section{Acknowledgement}

The authors gratefully acknowledge Dr. Mohammed Aman, Asst. Professor, Department of Microbiology, Jain University, Bengaluru for identifying the fungal species.

\section{Conflict of interest}

There is no conflict of interest for any of the authors.

\section{Bibliography}

1. Smil V. "Feeding the world: a challenge for the twenty-first century". Cambridge: The Massachusetts Institute of Technology Press (2000).

2. United Nations, Department of Economic and Social Affairs (2011).

3. Ingram J. "A food systems approach to researching food security and its interactions with global environmental change". Food Security 3 (2011): 417-431.

4. Savary S., et al. "Crop losses due to diseases and their implications for global food production losses and food security". Food Security 4 (2012): 519-537.

5. Adam Wightwick., et al. "Environmental Risks of Fungicides Used in Horticultural Production Systems". Fungicides, Odile Carisse (Ed.) (2010).

6. C Wang., et al. "Antifungal activity of eugenol against Botrytis cinerea". Tropical pathology 35.3 (2015): 137-143.

7. Olea., et al. "Antifungal Activity of Eugenol Derivatives against Botrytis Cinerea". Molecules 24 (2019): 1239.

8. Vinod Saharan., et al. "Synthesis and in vitro antifungal efficacy of Cu-chitosan nanoparticles against pathogenic fungi of tomato". International Journal of Biological Macromolecules 75 (2015): 346-353.
9. Plaza P., et al. "Evaluation of the potential of commercial postharvest application of essential oils to control citrus decay". The Journal of Horticultural Science and Biotechnology 76.6 (2004): 935-940.

10. W du Plooy., et al. "Essential oil amended coatings as alternatives to synthetic fungicides in citrus postharvest management". Postharvest Biology and Technology 53 (2009): 117122.

Volume 3 Issue 12 December 2019

(C) All rights are reserved by Sumangala Bhat K, et al. 